\title{
Biodegradable system for drug delivery of hydrolytically labile azanucleoside drugs
}

\author{
Martin Hruby ${ }^{a \#}$, Khushboo Agrawal ${ }^{b \#}$, Olivia Policianova a, Jiri Brus ${ }^{\mathrm{a}}$, Jan Skopal ${ }^{\mathrm{a}}$, Pavel Svec ${ }^{\mathrm{a}}$, Miroslav Otmarc, Petr Dzubak \\ Petr Stepanek ${ }^{a}$, Marian Hajduch ${ }^{b}$
}

Background. The archetypal DNA methyltransferase inhibitors, 5-azacytidine (AZA) and 5-aza-2'-deoxycytidine (DAC) are potent antineoplastic agents used in the treatment of mainly, blood malignancies. However, the administration of these drugs is confounded by their hydrolytic lability which decreases plasma circulation time. Here, we describe a new biodegradable, polyanhydride formulation for drug delivery that circumvents this drawback.

Methods. Injectable/implantable polymeric microbeads containing dispersed microcrystals of hydrophilic AZA or DAC packed in a dry environment are protected from hydrolysis, until the hydrolytic zone reaches the core. Diclofenac is embedded into the formulation to decrease any local inflammation. The efficacy of the formulations was confirmed by monitoring the induced demethylation, and cytostatic/cytotoxic effects of continuous drug release from the timecourse dissolution of the microbeads, using an in vitro developed cell based reporter system.

Results. Poly(sebaccic acid-co-1,4-cyclohexanedicarboxylic acid) containing 30 wt. \% drug showed zero-order release $\left(R^{2}=0.984\right.$ for linear regression), and release rate of $10.0 \% / h$ within the first $5 \mathrm{~h}$, and subsequent slower release of the remaining drug, thus maintaining the level of drugs in the outer environment considerably longer than the typical plasma half-life of free azanucleosides. At lower concentrations, the differences between powder drug formulations and microbeads were very low or negligible, however, at higher concentrations, we discovered equivalent or increasing effects of the drugs loaded in microbeads.

Conclusions. The study provides evidence that microbead formulations of the hydrolytically labile azanucleoside drugs could prevent their chemical decomposition in aqueous solution, and effectively increase plasma circulation time.

Key words: 5-azacitidine, 5-aza-2'-deoxycytidine, diclofenac, polyanhydride, microbeads

Received: July 23, 2015; Accepted with revision: March 3, 2016; Available online: March 21, 2016 http://dx.doi.org/10.5507/bp.2016.013

aInstitute of Macromolecular Chemistry, Academy of Sciences of the Czech Republic, v.v.i., Heyrovsky Sq. 2, 16206 Prague 6, Czech Republic ' Institute of Molecular and Translational Medicine, Faculty of Medicine and Dentistry, Palacky University Olomouc, Hnevotinska 5, 77900 Olomouc, Czech Republic

Institute of Organic Chemistry and Biochemistry, Academy of Sciences of the Czech Republic, v.v.i., Fleming Sq. 2.166 10 Prague 6, Czech Republic

\#These authors contributed equally to this work

Corresponding author: Marian Hajduch, e-mail: marian.hajduch@upol.cz

\section{INTRODUCTION}

Cytosine analogues, 5-azacytidine (azacytidine, AZA, Vidaza ${ }^{\circledR}$ ) and 5-aza-2'-deoxycytidine (decitabine, DAC, Dacogen ${ }^{\circledR}$ ) have been established as efficient therapeutics for the treatment of blood malignancies, especially myelodysplastic syndrome ${ }^{1-3}$. They have two mechanisms of action: at low doses, they inhibit the expression of de novo DNA methyltransferases (DNMTs), and reactivate the tumor suppressor genes silenced by aberrant DNA methylation. This determines the therapeutic efficacy of the drugs ${ }^{1-3}$. However, at high doses, they directly incorporate into nucleic acids resulting in impaired DNA and RNA synthesis, inhibition of protein production, and cell death. The effect on DNMTs is based on the nucleophilic attack of the azanucleosides on the carbon- 6 atom of the cytosine ring, resulting in covalent bond formation between azacytosine and the active site of the enzyme ${ }^{4}$. This covalent trapping of DNMTs is stable and inhibition is therefore practically irreversible. However, the susceptibil- ity of the carbon 6 of the azacytidine ring in the presence of other nucleophiles including water causes rapid and reversible opening of the 5-azacytosine ring, followed by irreversible decomposition. This hydrolytic lability renders the chemical instability of the azanucleoside drugs, thereby compromising the plasma circulation time ${ }^{5}$. Often, long-term cooled infusions are necessary. This underlines the paramount importance for the AZA and DAC formulations that may overcome this hydrolytic lability.

Biodegradable polymeric implants are well established delivery devices for various drugs. Drug release may be driven either by diffusion of the drug from the polymeric depot, cleavage of the chemical bond between polymeric carrier and the drug, degradation of the polymeric implant, or by combination of these mechanisms ${ }^{6-8}$. Besides, controlled chemical degradation of the implant forming polymer (desirable for many in vivo applications) may proceed in whole volume, e.g., hydrazone based hydrogels $^{9}$, from the surface only, e.g., polyanhydrides ${ }^{10-12}$, or by combination of both, e.g., polylactides ${ }^{8,13}$. 
Polyanhydrides such as poly(sebacic acid) are relatively hydrophobic polymers that degrade in aqueous milieu, by hydrolysis of carboxylic acid anhydride bond into low molecular weight water-soluble dicarboxylic acids which are further metabolized or excreted ${ }^{10,11,13}$. Since, hydrolysis of polyanhydrides strictly begin at the surface so the inner volume of the implant remains dry, until the hydrolytic zone on the surface reaches completely to the core. Rate of hydrolysis is inversely proportional to the hydrophobicity of the monomer (increased hydrophobicity decreases degradation rate), and can be controlled upon modification with highly hydrophobic fatty acids ${ }^{14}$, or with use of monomeric composition that increases crystallinity, such as conformationally rigid cyclic diacid comonomers (eg., 1,4-cyclohexanedicarboxylic acid), or aromatic diacid monomers ${ }^{10-12}$.

This study describes a conceptually new system exploiting the ability of the polyanhydrides to hydrolyze from the surface only, to release the hydrolytically labile drugs, AZA and DAC. Instead of hydrophobic polyanhydride, the injectable/implantable polymeric microbeads are biodegradable, containing dispersed microcrystals of hydrophilic AZA or DAC, insoluble in anhydride. With the time-course degradation of the polyanhydride, the degradation zone/aqueous milieu proceeds from the surface to the core of the beads, resulting in dissolution of microcrystals and sustained release of the drugs into outer environment. The underlining principle is based on the packing of microcrystals in a dry environment protected from hydrolysis, until the hydrolytic zone reaches the core, thus circumventing the hydrolytic lability (Fig. 1).

For preparation of the drug loaded microbeads, Poly(sebaccic acid-co-1,4- cyclohexanedicarboxylic acid) was used as the starting polymer. Since, microbeads may cause local irritation resulting in inflammation, nonsteroidal anti-inflammatory drug, diclofenac (DIC) was added to the polymeric matrix ${ }^{15}$. DIC is hydrophobic and is soluble in polymeric matrix.

This biodegradable model of micro-particulate dual drug delivery system was characterized in vitro for physical, chemical, and biological properties.

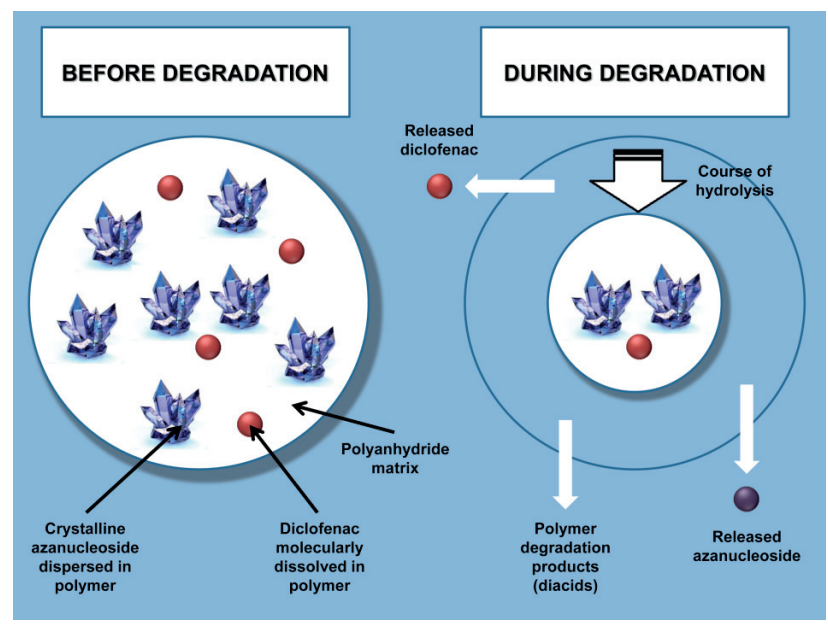

Fig. 1. Scheme of the microbead system.

\section{MATERIALS AND METHODS}

All chemicals were obtained from Sigma-Aldrich, St. Louis, MO, unless otherwise indicated.

\section{Preparation of polymeric beads}

For the preparation of Poly(sebaccic acid-co-1,4-cyclohexanedicarboxylic acid), PSA-co- PCH (70:30 mol $/ \mathrm{mol})$, a modified procedure described previously ${ }^{16}$ was used.

Sebaccic acid (8.40 g, $41.5 \mathrm{mmol}), 1$,4-cyclohexanedicarboxylic acid (3.06 g, $17.8 \mathrm{mmol})$ and acetic anhydride (115 mL, $124 \mathrm{~g}, 1210 \mathrm{mmol})$ were refluxed for $30 \mathrm{~min}$, and the reaction mixture was evaporated in vacuo. Subsequently, the residues of acetic acid and acetic anhydride were azeotropically removed by evaporation with toluene $(2 \mathrm{x})$, and the solid residue was dissolved in chloroform, followed by precipitation with diethyl ether - petroleum ether mixture $(1: 1 \mathrm{v} / \mathrm{v})$. The precipitated prepolymer was then filtered off, air-dried, and heated on magnetic stirrer at $180{ }^{\circ} \mathrm{C}$ for $90 \mathrm{~min}$ in vacuo $(10 \mathrm{~Pa})$, and cooled. Finally, the solidified melt was dissolved in dichloromethane and precipitated with petroleum ether, yielding $7.79 \mathrm{~g}$ (75\%) of purified PSA-co-PCH [Weightaverage molecular weight (Mw): $12.1 \mathrm{kDa}$, according to gel permeation chromatography], refer ss NMR characterization.

AZA and DAC, or uridine (model for in vitro release experiment) were homogenized in acetonitrile suspension (IKA T25 Ultra Turrax ${ }^{\circledR}$ dispersing instrument, Thermo Fisher Scientific Inc., Waltham, MA), filtered off, airdried, and milled (Pulverisette 23® Mini Mill, Fritsch, ILABO Ltd, Kyjov, Czech Republic). PSA-co-PCH (420 $\mathrm{mg}$ ) was dissolved in anhydrous acetonitrile $(1.78 \mathrm{~mL})$ at $60{ }^{\circ} \mathrm{C}$ and milled AZA, DAC or uridine (180 mg) were added subsequently. For dispersions containing DIC, the amount of PSA-co-PCH was reduced to $360 \mathrm{mg}$ and DIC $(60 \mathrm{mg})$ was added. The suspension $\left(58^{\circ} \mathrm{C}\right)$ was emulsified with $50 \mathrm{~mL}$ of polyisobutylene thickened mineral oil $\left(58{ }^{\circ} \mathrm{C}\right.$ ) by vigorous stirring at $2000 \mathrm{rpm}$, and the resulting emulsion was stirred at $60{ }^{\circ} \mathrm{C}$ for $30 \mathrm{~min}$, until complete evaporation of the acetonitrile. The suspension was cooled to room temperature with stirring, and filtered. Finally, the beads were collected and washed several times with hexane to get rid of mineral oil and polyisobutylene, air-dried, and washed again quickly with $\mathrm{H}_{2} \mathrm{O}$ to remove surface- bound drug crystals, and immediately dried in vacuo. Bead size distribution, weighted by volume fraction was determined by Mie scattering (Mastersizer 3000 instrument, Malvern Instruments Ltd, United Kingdom).

\section{In vitro drug release}

Uridine was used as a model compound for release of AZA or DAC from the beads due to chemical stability, structural similarity, and analytical simplicity. Beads with 10, 20 and 30 wt. \% uridine (absorbance ca $\mathrm{A}_{\max }=1.000$ for $100 \%$ release, extinction coefficient $\varepsilon_{261 \mathrm{~nm}}=1.197 \times 104$ $\mathrm{L} / \mathrm{mol} / \mathrm{cm}$ ) were suspended in $200 \mathrm{~mL} \mathrm{PBS} \mathrm{(pH} \mathrm{7.4),} \mathrm{and}$ incubated with shaking at $37^{\circ} \mathrm{C}$. Samples (aa $0.5 \mathrm{~mL}$ ) were filtered and absorbance $A$ was measured at $261 \mathrm{~nm}$ against PBS at selected time points. Relative release was 
calculated by assuming uridine as the only UV-absorbing component (verified by experiment with "empty" PSAco-PCH beads without uridine), and neglecting sampling volume change (aa $0.5 \mathrm{~mL}$ vs $200 \mathrm{~mL}$ ), according to equation, $\mathrm{R}=\left(A / A_{\max }\right) * 100 \%$.

\section{Solid state nuclear magnetic resonance (ss NMR) characterization of the beads}

All solid state NMR spectra were measured at $11.7 \mathrm{~T}$ (Bruker Avance III HD 500 US/WB NMR spectrometer, Bruker, Karlsruhe, Germany) in 3.2-mm ZrO2 rotors, at spinning frequency of $20 \mathrm{kHz}$. The ${ }^{13} \mathrm{C}$ cross-polarization magic angle spinning NMR spectra $\left({ }^{13} \mathrm{C} \mathrm{CP} / \mathrm{MAS} \mathrm{NMR}\right)$ were measured with $\mathrm{CP}$ contact time of $2 \mathrm{~ms}$, repetition delay of $5 \mathrm{~s}$, and number of scans ranging from 1024 to 2048, to reach acceptable signal to noise ratio. However, the ${ }^{13} \mathrm{C}$ MAS NMR spectra with direct excitation of ${ }^{13} \mathrm{C}$ magnetization were recoded using an excitation of 90 degree, ${ }^{13} \mathrm{C}$ pulse of $2 \mu \mathrm{s}$, repetition delay of $10 \mathrm{~s}$ and 3600 scans, to reach the acceptable signal to noise ratio. During the detection of ${ }^{13} \mathrm{C}$ NMR signal, the high power dipolar decoupling, SPINAL-64 was applied, and the frictional heating of the spinning samples was mitigated by active cooling ${ }^{17}$. The temperature calibrations were performed with $\mathrm{Pb}(\mathrm{NO} 3)_{2}$, and glycine was used as an external standard to calibrate the ${ }^{13} \mathrm{C}$ scale (176.03 ppm - low-field carbonyl signal).

\section{In vitro biological evaluation}

The demethylation and cytostatic/cytotoxic effects of the drug loaded microbeads were characterized in vitro, using the demethylation reporter cell line (HCT116-pFLJH2B), developed and validated in our laboratories. The colorectal cancer cell line, HCT116 was stably transfected with the hypermethylated promoter, FLJ32130 tagged with EGFP, for reporting demethylation drug effects ${ }^{18}$, and further transduced with histone $2 \mathrm{~B}$ tagged with RFP, to stain the cell nuclei (LentiBrite ${ }^{\mathrm{TM}}$ Histone H2B-RFP Lentiviral Biosensor, EMD Millipore, Billerica, MA) for continu- ous monitoring of cell counts, reflecting the cytostatic/ cytotoxic drug effects.

Using this reporter cell line, demethylation activity of the drugs can be assayed by quantifying the intensity of EGFP fluorescence following drug treatment. The intensity of EGFP fluorescence is directly proportional to demethylation potential of the drug, however, in untreated cells EGFP fusion protein would not be expressed. Likewise, cytostatic/cytotoxic drug effects can be monitored by counting the cell numbers in untreated and experimental wells, under RFP channel, utilizing continuous live cell fluorescent imaging (Fig. 2).

\section{Cell Culture and Drug Treatment:}

The reporter cell line was cultured in McCoy's 5A medium, supplemented with $10 \%$ fetal bovine serum (PAN-Biotech GmbH, Aidenbach, Germany), $3 \mathrm{mM}$ L-glutamine, $50 \mu \mathrm{g} / \mathrm{mL}$ streptomycin and $100 \mathrm{U} / \mathrm{mL}$ penicillin (Biotika, Slovenská Lupča, Slovak Republic), at $37{ }^{\circ} \mathrm{C}$ and $5 \% \mathrm{CO}_{2}$ in a humidified incubator. On day 0 , the cells were plated at the density of $2 \times 10^{4}$ cells per $3.8 \mathrm{~cm}^{2}$ well on 6 well glass bottom plate (P06-1.5H-N, In Vitro Scientific, Sunnyvale, CA). After $24 \mathrm{~h}$, drugs in powder formulations or microbeads were added in three different concentrations, viz. $25 \mu \mathrm{mol} / \mathrm{L}, 50 \mu \mathrm{mol} / \mathrm{L}$ and $100 \mu \mathrm{mol} / \mathrm{L}$. For powdered formulation of the drugs, 50 $\mathrm{mM}$ solution was freshly prepared in DMSO (the final concentration of DMSO was below $0.1 \%$ ), however, in case of drugs loaded in microbeads, weight equivalents of the solid microbeads were directly added to well plates, and volume of the culture medium was adjusted to maintain the required drug concentration. Untreated wells were marked as control for the baseline measurement.

\section{High Content Cellular Imaging and Data Analysis:}

For monitoring the induced effects of the continuous drug release from the time-course dissolution of the microbeads in culture medium, cellular images were acquired at every $24 \mathrm{~h}$ intervals following treatment, from day 1 to day
A.

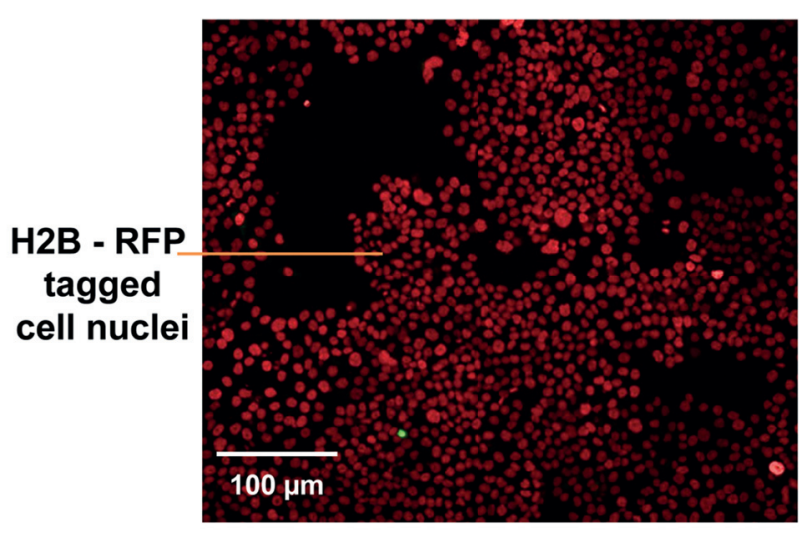

Control
B.

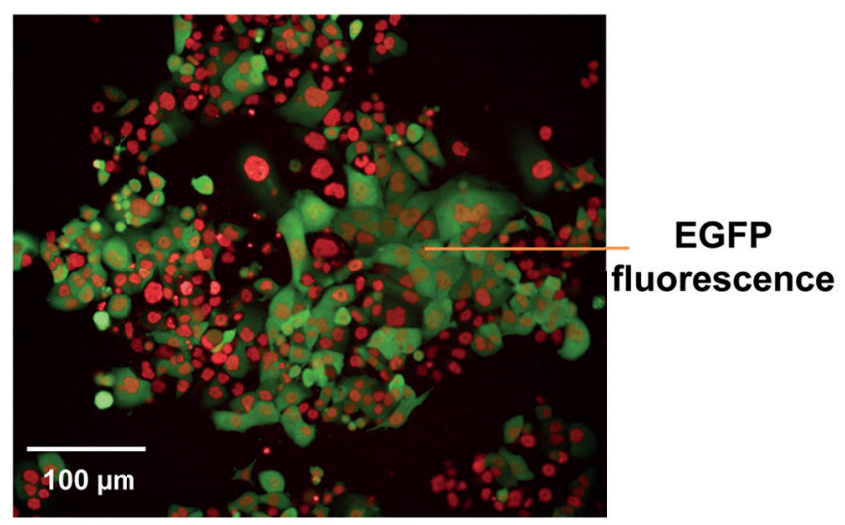

Treated

Fig. 2. Pictorial illustration of high content image analysis. [A] Untreated cells showing RFP nuclear fluorescence but no EGFP expression due to hypermethylated promoter. [B] Anticipated EGFP fluorescence following treatment with DNA methylation inhibitor, indicating promoter demethylation. Cytotoxicity was determined by counting the total number of cell nuclei (red, RFP), and demethylation was inferred by quantifying EGFP fluorescence (green). Objective: 20×, Scale bar - $100 \mu \mathrm{m}$. 
6, under wavelengths appropriate for excitation/emission (EGFP: $458 \mathrm{~nm} / 525 \mathrm{~nm}$, RFP: $555 \mathrm{~nm} / 584 \mathrm{~nm}$ ), utilizing high content imaging system (Operetta, PerkinElmer, Waltham, MA). Images were analyzed using image analysis software (Columbus, PerkinElmer). Fig. 2, exemplify the pictorial diagram for high content image analysis.

For reporting demethylation, the analysis was interpreted as mean EGFP intensity per cell, calculated by dividing mean EGFP intensity per well with total number of cells in each well. However, the total number of cells counted under RFP channel was used to report the cytostatic/cytotoxic drug effects. At the end, EGFP fluorescence intensities measured from day 1 to day 6 were integrated to calculate area under the curve (AUC) for demethylation, for each drug concentration separately. Similarly, total number of cells counted from day 1 to day 6 was integrated to calculate the AUC for cytotoxicity (GraphPad Prism version 6.01, GraphPad Software Inc., La Jolla, CA).

\section{[A]}

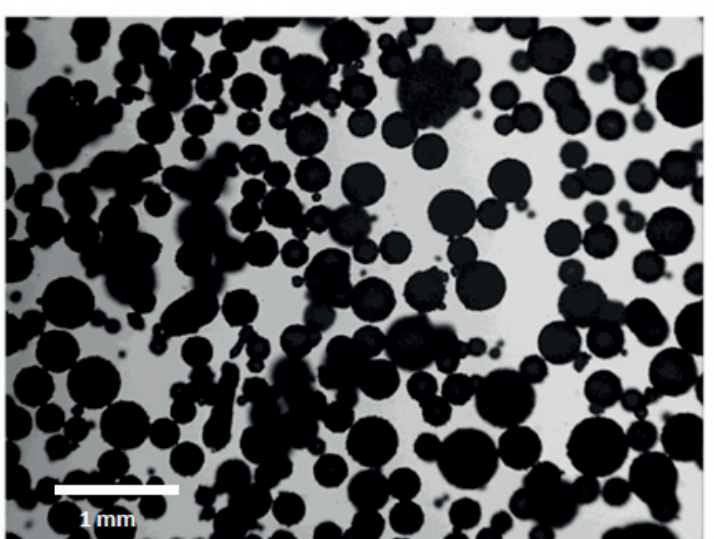

[C]

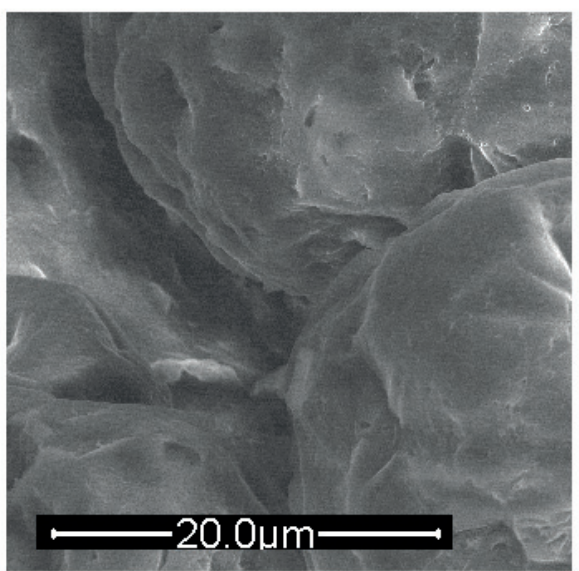

\section{RESULTS AND DISCUSSION}

\section{Preparation of microbeads and characterization of} in vitro release rate

The beads were prepared by solvent evaporation method. The polyanhydride, and/or DIC were dissolved in dry acetonitrile, and finely milled AZA or DAC (nearly insoluble in acetonitrile) were suspended subsequently. The suspension was emulgated in mineral oil thickened with polyisobutylene, to suppress the microparticle aggregation, which is immiscible with acetonitrile and does not dissolve AZA, DAC, DIC or polyanhydride. The solvent (acetonitrile) was evaporated and droplets were formed into beads with desired inner architecture of microcrystals embedded in spherical matrix, formed by polymer or polymer with dissolved DIC. The average size of the microparticles was $300 \mu \mathrm{mol} / 1$ in all cases, see Fig.3A for typical micrograph.

Different formulations of azanucleosides prepared in microbeads were 5\% DAC, 5\% AZA, 30\% DAC, and 30\% $\mathrm{DAC}+10 \%$ DIC

To characterize the in vitro release rate of azanucleosides, uridine was used as the model, due to its similar

\section{[B]}

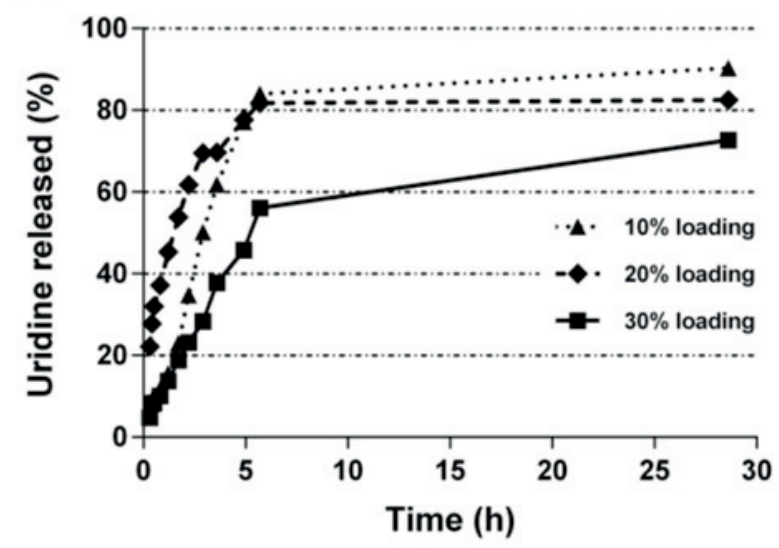

[D]

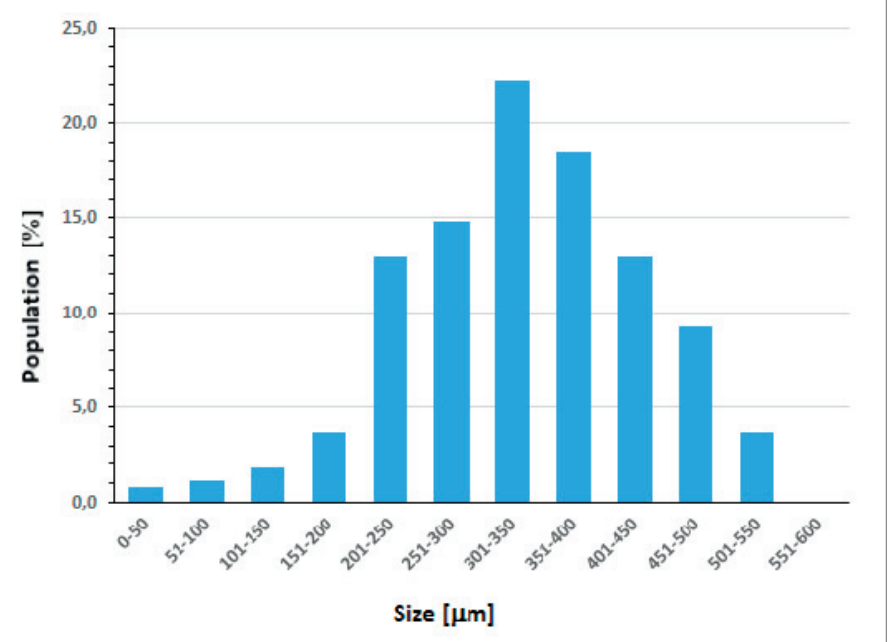

Fig. 3. [A] The polymer beads with embedded AZA crystals. Magnification: 10×, bar $=1 \mathrm{~mm}$. [B] In vitro release of 10, 20, and 30 wt. \% uridine loadings from the beads into PBS ( $\mathrm{pH} 7.4)$ at $37{ }^{\circ} \mathrm{C}$. [C] Surface of the microparticles under scanning electron microscopy. [D] Typical histogram of the size distribution of the microparticles, weighted by volume fraction (Mie scattering). 
physical and chemical properties with AZA and DAC, and chemical stability, unlike hydrolytically labile AZA and DAC (ref. ${ }^{5}$ ). Besides, at $261 \mathrm{~nm}$, uridine is only UVabsorbing component in uridine-polyanhydride system, both before and after degradation.

Initially, beads were incorporated with five different loadings of uridine viz., 10, 20, 30, 40 and 50 wt. \% of beads respectively. However, maximal loading to keep the microparticles cohesive was $30 \mathrm{wt}$. \%, and loadings of 40 and $50 \mathrm{wt}$. \% were not coherent, so only beads with 10, 20 and $30 \mathrm{wt} . \%$ uridine were tested for release.

Further, as seen in Fig. 3B, formulations with 10 and 20 wt. \% loading showed relatively fast release within first $5 \mathrm{~h}$, until the release of ca $80 \%$ uridine, followed by significant slower release. Adversely, beads with 30 wt. \% loading released uridine more slowly amongst all with zero order kinetics $\left(\mathrm{R}^{2}=0.984\right.$ for linear regression $)$, and the release rate of $10.0 \% / \mathrm{h}$ within first $5 \mathrm{~h}$, followed by significant slower release. The beads with $30 \mathrm{wt}$. \% loading were therefore able to maintain the level of drugs in outer environment, considerably longer than the typical plasma half-life of free azanucleosides.

Surface of the microparticles (Fig. 3C) as well as their size distribution (Fig. 3D), both may have influence on the release rate. In accordance with the release data, surface of the microparticles is smoothly and continuously covered with polymer, sufficient to provide defined release rate from the beginning. On the other hand, the microparticles are somehow polydisperse in size (Fig. 3D), but with negligible effect on the release rate (Fig. 3B).

Release of DIC from the beads was also confirmed by the test (data not shown), but experiment was complicated by the limited solubility of DIC in the concentrations suitable for " $100 \%$ release" of azanucleosides ${ }^{15}$.

\section{Structural ss-NMR analysis}

For structural confirmation of the particles, dispersion with $30 \mathrm{wt} \%$ DAC and 10 wt. \% DIC was chosen for further study by solid state NMR spectroscopy. Interpretation of the recorded data is based on previous comprehensive structural ss-NMR analyses of various polymeric solid dispersions of active compounds ${ }^{19-20}$, see Fig. 4 for the chemical structures.

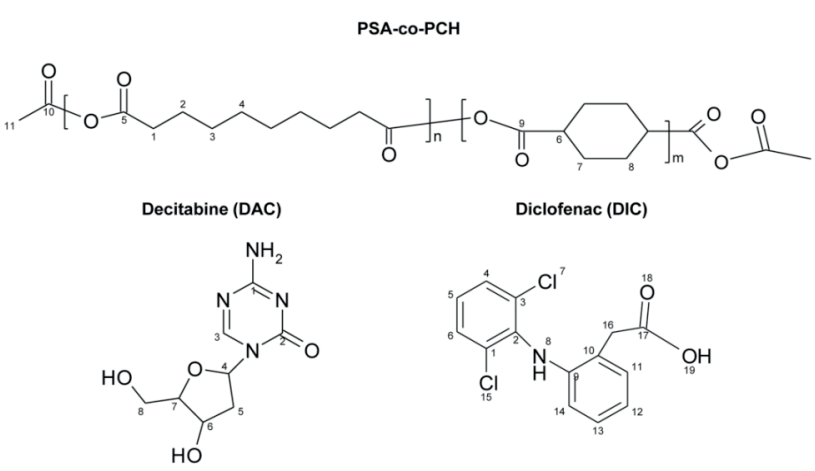

Fig. 4. Chemical structures of the components. The copolymer, PSA-co-PCH, and the active pharmaceutical ingredients, DAC and DIC.
The narrow well-resolved signals detected in ${ }^{13} \mathrm{C} \mathrm{CP} /$ MAS NMR spectra of powdered DAC and DIC (Fig. 5A and $5 \mathrm{~B}$ respectively) indicated a single-phase highly crystalline character of the active pharmaceutical ingredients (APIs), used for the preparation of solid dispersions. In contrast, the structure of PSA-co-PCH copolymer matrix was far complicated, as followed from the comparison of ${ }^{13} \mathrm{C} \mathrm{CP} / \mathrm{MAS}$ and ${ }^{13} \mathrm{C}$ MAS NMR spectra (Fig. 5C and $5 \mathrm{D}$ respectively), optimized for selective measurements of rigid (crystalline), and mobile (amorphous) phases respectively. While the ${ }^{13} \mathrm{C} \mathrm{CP/MAS} \mathrm{NMR} \mathrm{spectrum}$ of PSA-co-PCH copolymer was dominated by the set of strong narrow lines at 168, 36, 34, 33 and 25 ppm, attributed to highly crystalline PSA block (carbons 5, 1, 4, 3, 2 respectively), the ${ }^{13} \mathrm{C}$ MAS NMR spectrum consisted of the narrow signals at 170, 35, 29, 28 and 24 ppm, corresponding to highly mobile PSA segments in amorphous phase (carbons 5, 1, 4, 3, 2 respectively). Furthermore, the presence of immobilized amorphous PSA blocks was indicated by a weak broad signal at ca. 29 ppm, and presence of amorphous PCH segments was indicated by the weak signals at ca. 41 and $27 \mathrm{ppm}$, corresponding to the carbons 6 and 7, 8 respectively, recorded in ${ }^{13} \mathrm{C} \mathrm{CP} / \mathrm{MAS}$ NMR spectrum (Fig. 5C). Besides, the weak signals, particularly detected in ${ }^{13} \mathrm{C}$ MAS NMR spectrum (Fig. 5D) correspond to monomer units that couple PSA and $\mathrm{PCH}$

[A]

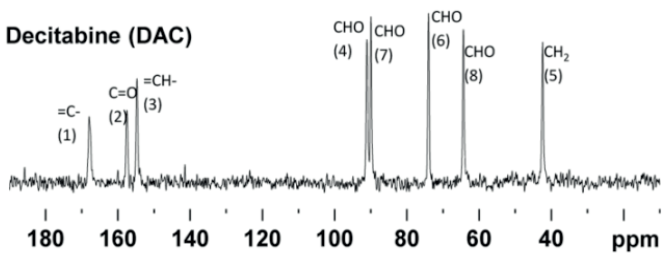

Diclofenac (DIC)

[B]

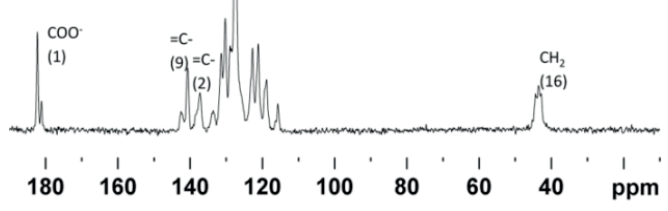

[C]
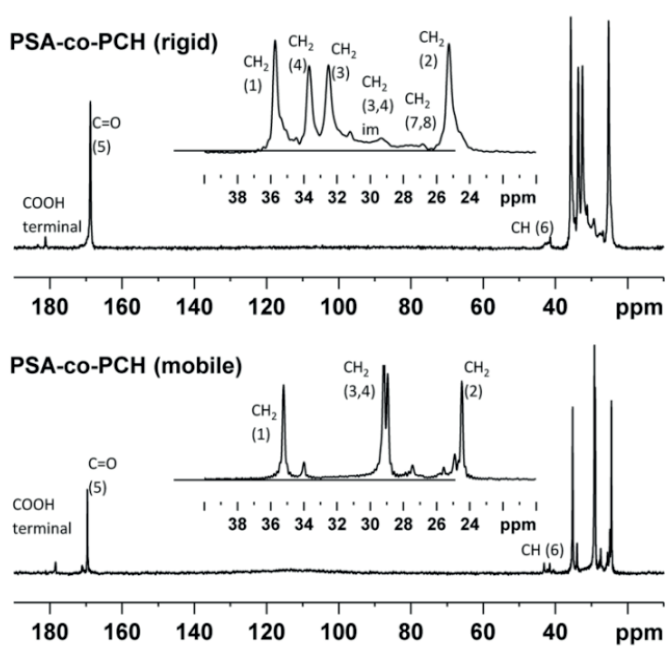

Fig. 5. The ${ }^{13} \mathrm{C} \mathrm{CP} / \mathrm{MAS}$ NMR spectra of [A] Decitabine, [B] Diclofenac and [C] PSA-co-PCH copolymer, and [D] the ${ }^{13} \mathrm{C}$ MAS NMR spectrum of the PSA-co-PCH copolymer. 
blocks. These spectra thus clearly reflect the heterogeneous character of the PSA-co-PCH copolymer that consists of both, rigid (crystalline) and mobile (amorphous) phase of PSA blocks, and PCH segments that are spread in both phases (rigid and mobile). Overall, it is clear that structure of the prepared solid dispersions, DAC/PSA-coPCH, DIC/PSA-co- PCH, and DAC+DIC/PSA-co-PCH are hardly predictable.

\section{ss NMR, characterizing the distribution of APIs in copolymer matrix}

The recorded ${ }^{13} \mathrm{C} \mathrm{CP} / \mathrm{MAS}$ and ${ }^{13} \mathrm{C}$ MAS NMR spectra of the solid dispersions further revealed the considerable differences in the distribution of DIC and DAC in the copolymer matrix.

Specifically, the set of narrow and well-resolved signals of DAC were clearly detected in ${ }^{13} \mathrm{C} \mathrm{CP} / \mathrm{MAS}$ NMR spectrum. The resonance frequencies of these signals were identical with the resonance frequencies recorded for pure, crystalline DAC, indicating unchanged polymorphic form of DAC. In contrast, DAC signals were absent in corresponding ${ }^{13} \mathrm{C}$ MAS NMR spectrum, measured with a relatively short repetition period. These facts indicated that in DAC/PSA-co-PCH solid dispersion, the molecules of DAC were partly phase-separated, forming nano crystalline domains. Moreover, preliminary ${ }^{13} \mathrm{C}$ detected $\mathrm{T}_{1}\left({ }^{1} \mathrm{H}\right)$ relaxation experiments showed incomplete ${ }^{1} \mathrm{H}$ polarization transfer between crystalline DAC and crystalline fraction of PSA, indicating the close contact of these fractions. Thus, crystallites of decitabine are probably surrounded by the crystalline fraction of PSAco-PCH copolymer (Fig. 6A).

In contrast to DAC/PSA-co-PCH solid dispersion, the signals of the active compound (DIC) in DIC/PSA-co$\mathrm{PCH}$ system were detected only in single-pulse ${ }^{13} \mathrm{C}$ MAS NMR spectrum, while in ${ }^{13} \mathrm{C} \mathrm{CP} / \mathrm{MAS}$ NMR spectrum, only the signals of crystalline rigid fraction of PSA blocks were identified. Thus, the presence of extremely narrow signals of DIC $(<35 \mathrm{~Hz})$ in ${ }^{13} \mathrm{C}$ MAS NMR spectrum, and the absence of the corresponding signals in ${ }^{13} \mathrm{C} \mathrm{CP} / \mathrm{MAS}$ NMR spectrum, confirmed nearly unrestricted molecular motion of DIC molecules in DIC/PSA-co-PCH solid dispersion. Consequently, it can be assumed that the active compound (DIC) is molecularly dispersed (dissolved) in highly mobile, amorphous fraction of PSA-co-PCH matrix (Fig. 6B).

However, the most complicated spectra were recorded for the triple-component solid dispersion, DAC+DIC/ PSA-co-PCH, where both the active compounds incorporated in PSA- co-PCH copolymer matrix were detected in ${ }^{13} \mathrm{C}$ MAS NMR spectrum (red line) as broad unresolved signals, whereas, the corresponding signals were not detected in ${ }^{13} \mathrm{C} \mathrm{CP} / \mathrm{MAS}$ NMR spectrum (blue line). The observed broadening of the signals in ${ }^{13} \mathrm{C}$ MAS NMR spectrum, and the absence of the signals in ${ }^{13} \mathrm{C} \mathrm{CP} / \mathrm{MAS}$ NMR spectrum thus indicated considerably disordered
[A]

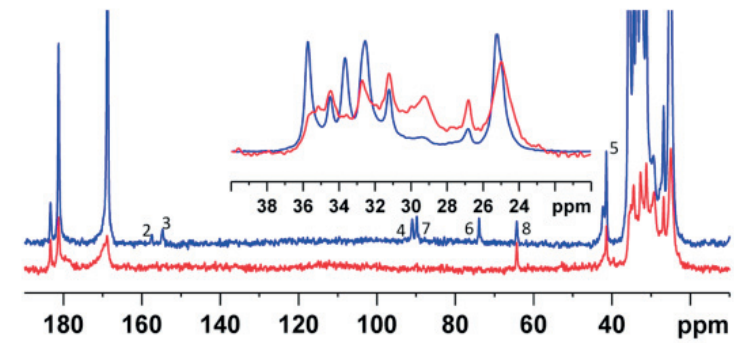

[B]

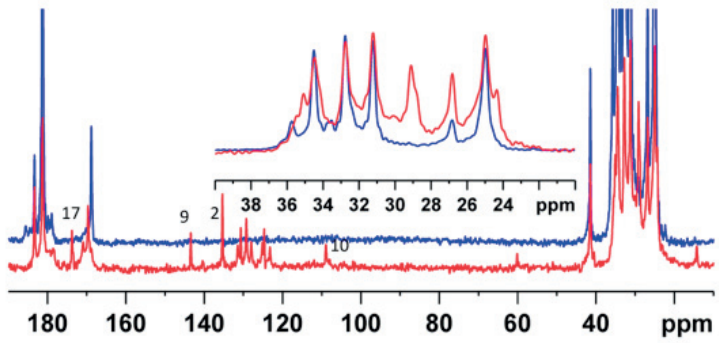

[C]

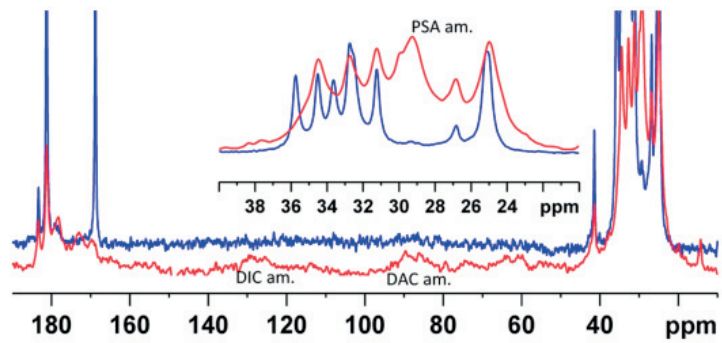

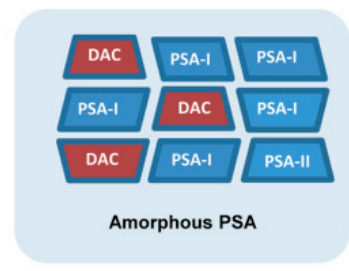

DAC/PSA-CO-PCH

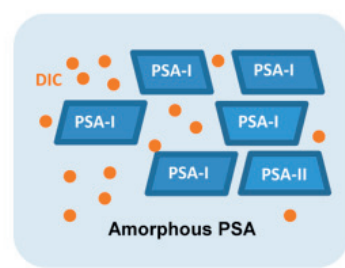

DIC/PSA-Co-PCH

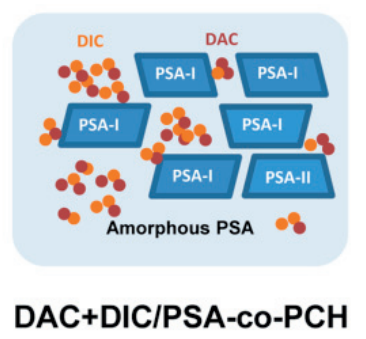

Fig. 6. The ${ }^{13} \mathrm{C} \mathrm{CP} / \mathrm{MAS}$ (blue lines) and ${ }^{13} \mathrm{C}$ MAS (red lines) NMR spectra, and the schematic structural representation of the solid dispersions. [A] DAC/PSA-co-PCH, [B] DIC/PSA-co-PCH and $[\mathrm{C}]$ DAC+DIC/PSA-co-PCH. 
and amorphous character of both APIs. In consistence with the molecular mobility, the molecules of DAC and DIC probably exhibit restricted high- amplitude motions. On one hand, the motion is sufficiently fast to destroy the static ${ }^{1} \mathrm{H}-{ }^{13} \mathrm{C}$ dipolar couplings, disallowing the detection of the cross-polarization signals. On other hand, the motion is too slow to completely remove the signal broadening, resulting from the conformational and orientational non-equivalence of the molecules of APIs in amorphous phase. This further indicates the weak interactions between APIs and the polymer matrix. Thus, molecules of APIs are either dispersed in the amorphous, semi-flexible phase of PSA, and/or form nano-size clusters. For discrimination of these possibilities, more extensive experimentation is required. However, the detailed structural analysis of the prepared multicomponent systems, currently under progress, is beyond the scope of this literature (Fig. 6C).

In conclusion, solid-state ${ }^{13} \mathrm{C}$ NMR experiments, optimized for domain-selective detection of the rigid, and/ or mobile fractions, enabled the primary characterization of the multicomponent solid dispersions of DAC and DIC, in semi-crystalline PSA-co-PCH copolymer matrix. Noticeably, three types of solid dispersions (DAC/PSA-co$\mathrm{PCH}$, DIC/PSA-co-PCH, and DAC+DIC/PSA-co-PCH), each exhibiting different molecular structures were analyzed. While, the DAC/PSA-co-PCH solid dispersion was distinguished as a partially phase-separated system with crystalline domains of DAC probably surrounded by the crystalline fraction of PSA blocks, the DIC/PSA-co-PCH system was basically homogenous with highly mobile molecules of DIC dispersed in flexible amorphous fractions of PSA chains. Whereas, the three-component solid dispersion, DAC+DIC/PSA-co-PCH exhibit far complicated structure in which both active compounds form amorphous semi- flexible phase dispersed in the copolymer matrix. Also, clustering of the molecules of APIs can be assumed.

\section{Biological interpretation of the therapeutic efficacy}

The time-course analysis of the observed biological effects of various formulated solid dispersions with different loadings of APIs viz., 5\% DAC, 5\% AZA, 30\% DAC, $30 \%$ DAC $+10 \%$ DIC, and powder formulations of the respective drugs (DAC and AZA), clearly demonstrated the exponential increase in the demethylation activity, in a time dependent manner until day 5 , after which begin the gradual decrease. Parallel was observed for cytotoxicity. On comparing the powder drugs with microbeads for demethylation activity, microbeads were less effective at $25 \mu \mathrm{mol} / \mathrm{L}$ concentration with low significant differences which started reducing at higher concentrations, and effect of microbeads was nearly equivalent or higher at $100 \mu \mathrm{mol} / 1$ concentration. In case of cytotoxicity, the differences between powder drugs and microbeads were almost negligible at $25 \mu \mathrm{mol} / \mathrm{L}$ and at higher concentrations, comparatively better results were observed for microbeads.

However, formulation with additional diclofenac $(30 \%$ DAC + 10\% DIC) showed least demethylation and cytotox- ic effects, indicating decreased drug efficacy for additional diclofenac. The decrease in hypomethylation efficacy in the presence of diclofenac may probably be a pharmacological interaction on cell- biochemical level, since the two molecules differ significantly in their physico-chemical interactions, under relevant conditions. However, after further optimization of the ratio (DAC:DIC), the effect of the anti- inflammatory drug, diclofenac is expected to remain local to the application site, as intended, while the effect of the hypomethylation drug, decitabine would be systemic (Fig. 7).

At last, considering the fact that the polymeric microbeads used for loading drugs may exhibit auto fluorescence or significant cytotoxicity, resulting in false interpretation of the results, beads without loaded DAC or AZA was also incorporated during the experiment (100\% polymer, $30 \%$ diclofenac, $5 \%$ uridine), but the values obtained were similar to the untreated cells (data not shown).

Besides, the present study was performed in cell culture system and it was difficult to analyze the demethylation profile after day 6 , due to increasing number of dead cells in experimental wells because of high drug concentrations used, and the confluency attained in control wells. Also, it was not suitable to perform the experiment using drug concentration lower than $25 \mu \mathrm{mol} / \mathrm{L}$ due to difficulty in weighing low amount of beads, and the concentrated molar solutions were not possible for this type of experiment. However, on the basis of microscopic observations, we assume that if the experiment is prolonged, the effects of microbeads will overcome the powder drug formulation, which is subjected to rapid chemical decomposition in aqueous solution after a short life span.

Moreover, the hypomethylation efficacy may also be influenced by the fact that the inhibition of DNMTs by azacytidine nucleosides is irreversible, and the turnover of the enzyme in a cell is relatively slow, so even a short exposition to free azanucleosides may have effect in vitro due to high concentrations, causing immediate distribution of the drug within the cultivation well. On contrary, sufficiently long exposition is required in vivo for proper exposition of all malignant cells within the organism.

\section{CONCLUSION}

In this study, we sought to elucidate the induced demethylation and cytotoxic effects of DAC and AZA loaded in biodegradable microbeads, in comparison with the powder formulations, and we report almost negligible or no differences between powder drug formulations and microbeads, at lower concentration, instead, at higher concentrations, we discovered equivalent or increasing effects of the drugs loaded in microbeads.

Besides, on comparing the microbeads formulations with different loadings of azanucleoside drug (DAC), no significant difference was observed. Therefore, it may be advantageous (as in most cases) to achieve as high drug loading as possible, without compromising the physicochemical and biological properties. This will significantly 


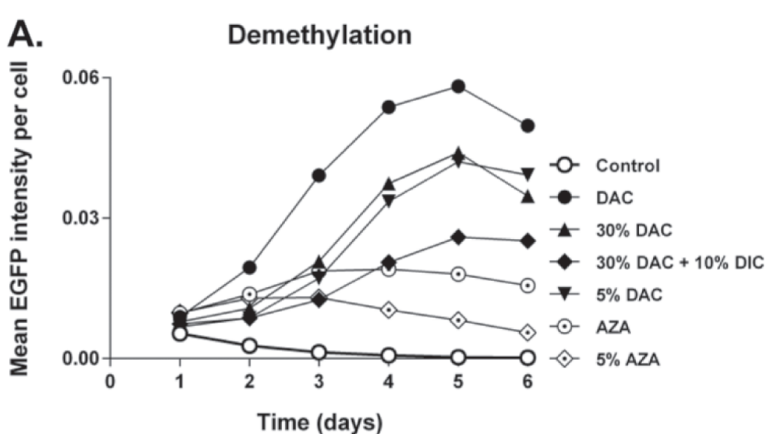

B.

$25 \mu \mathrm{mol} / \mathrm{L}$

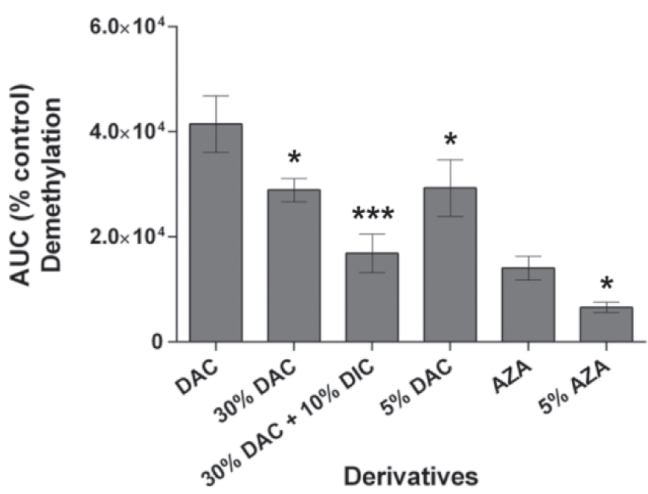

C.

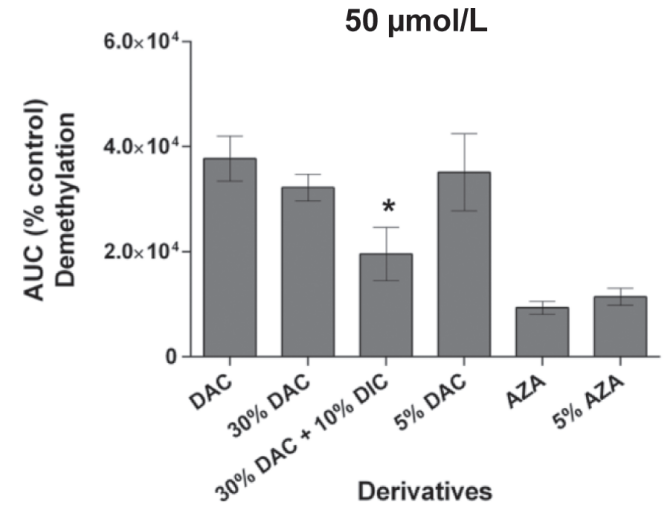

D.

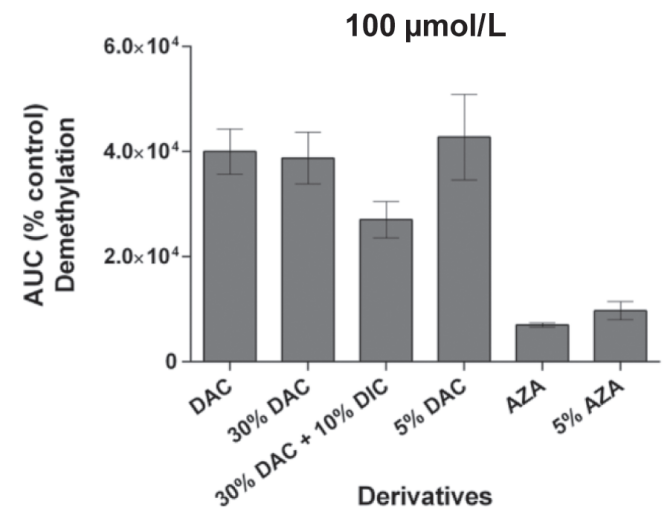

E.

Cytotoxicity

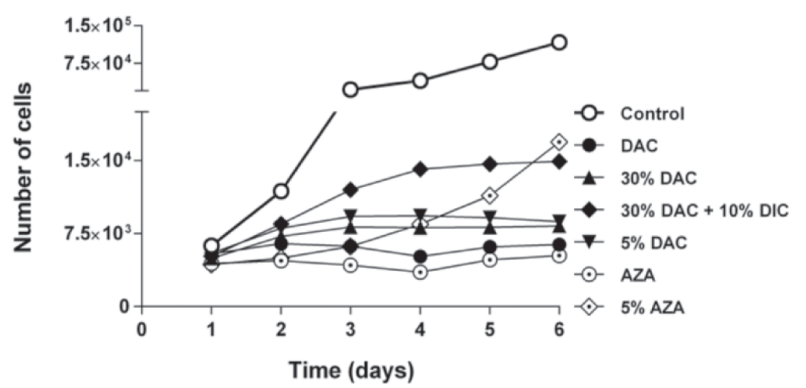

$25 \mu \mathrm{mol} / \mathrm{L}$

F.

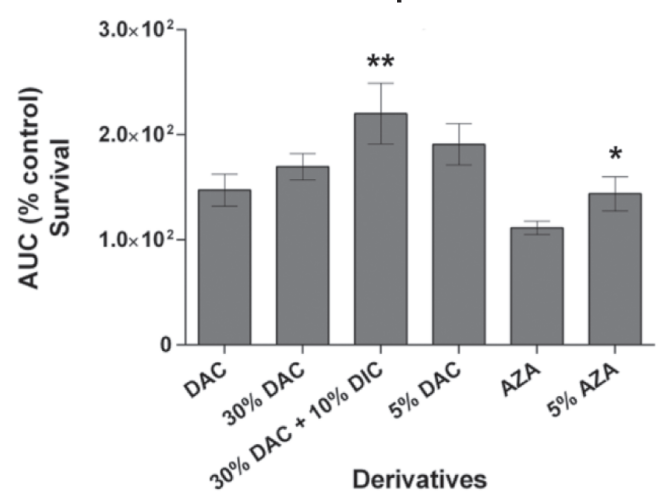

G.

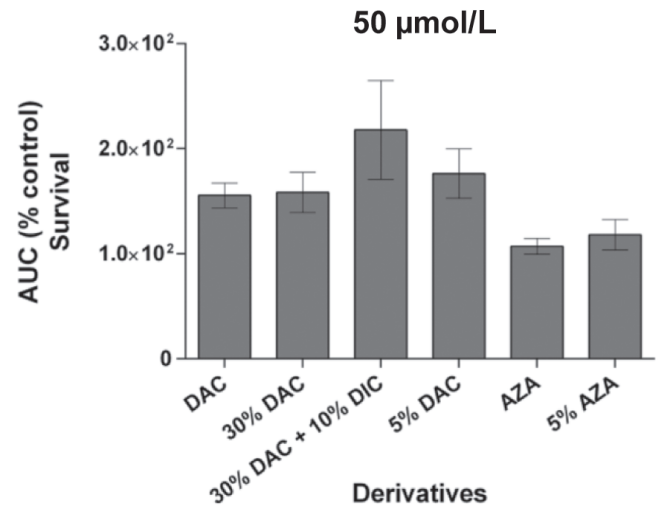

H.

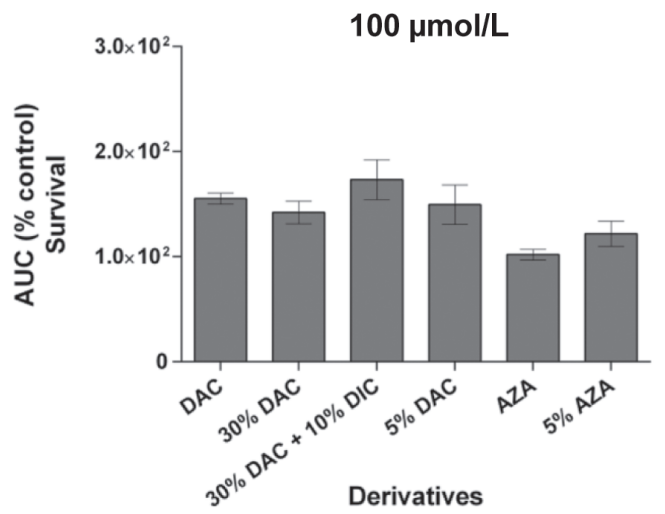

Fig. 7. Characterization of demethylation and cytotoxic effects induced by DAC and AZA: powder drug formulation vs microbeads. Demethylation [A], and Cytotoxicity [E], represent schematic plot for calculation of AUC at $25 \mu \mathrm{mol} / \mathrm{L}$, AUC for demethylation was calculated from the mean EGFP intensity per cell, quantified from day 1 to day 6, similarly, AUC for cytotoxicity was calculated using number of cells. [B - D], show AUC plots for demethylation, and [F - H], show AUC plots for cytotoxicity. Values are means \pm S.D,$n=3$. Statistical significance, ${ }^{*} P \quad 0.05, * * P \quad 0.005, * * * P \quad 0.0005$, was determined by comparing DAC, powder drug with its various formulations in microbeads, likewise, AZA, powder drug with its formulation in microbeads. At $25 \mu \mathrm{mol} / \mathrm{L}$, the microbeads were less effective with few significant differences, and at $50 \mu \mathrm{mol} / \mathrm{L}$ and $100 \mu \mathrm{mol} / \mathrm{L}$, almost no significant differences were observed, instead, microbeads seemed equally or more effective. 30\% DAC+10\% DIC was comparatively less effective than DAC. No statistical significance was determined on comparing the high drug loading (30\% DAC) with low drug loading (5\% DAC). 
reduce the amount of additional excipients and adjuvants (which should be eliminated from the organism after use), and also reduce the application volume for injection.

In conclusion, this study predicts that the substitution of the powder drug formulations of DAC and AZA, for injections, with drugs loaded microbeads, may increase the plasma circulation time of these hydrolytically labile drugs, and release the patients from the painful treatment therapy by reducing the number of intravenous injections per cycle of the treatment, on account of the chemical decomposition of the powder drug in aqueous solution.

To our knowledge, this is the first demonstration of the comparative effects of the powder, and the biodegradable microbeads formulations for the studied drugs in vitro, however, the complex preclinical data still needs to be determined.

\section{ABBREVIATIONS}

APIs, Active pharmaceutical ingredients; AUC, Area under the curve; AZA, 5-azacytidine; DAC, 5-aza-2'-deoxycytidine; DIC, Diclofenac; PSA-co-PCH, poly(sebaccic acid-co-1,4- cyclohexanedicarboxylic acid).

Acknowledgement: The authors are grateful to Dr. Toshikazu Ushijima, Carcinogenesis Division, National Cancer Center Research Institute for his kind gift of FLJ32130 targeting vector. The authors also thank to Dr. Miroslav Šlouf, Institute of Macromolecular Chemistry AS CR, v.v.i., for scanning electron microscopic measurment. This study was supported by the grants awarded by the Ministry of Industry and Trade of the Czech Republic (MPO TIP FR-TI4/625), Ministry of Education, Youth and Sports (CZ09/7F14009 and LO1304), Grant Agency of the Czech Republic (14-02652S and 14-03636S), and the Internal Grant Agency of Palacky University (IGA UP LF-2013/016).

Author contributions: $\mathrm{MHr}$ : designed the microbead system, synthesized the microbeads, and performed the drug release experiments; KA: determined the therapeutic efficacy of the microbeads in biological system, and drafted the manuscript; OP: conducted the solid state NMR studies; JB: designed the solid state NMR studies, and evaluated the ss NMR results; JS, PS: synthesized the microbeads, and performed the drug release experiments; MO: synthesized the azanucleosides; PD, PS: contributed in designing of the work, and data discussions; MHa: supervised the project, critically reviewed the data, and the manuscript.

Conflict of interest statement: The authors state that there are no conflicts of interest regarding the publication of this article.

\section{REFERENCES}

1. Bryan J, Kantarjian H, Jabbour E. Decitabine for the treatment of acute myeloid leukemia. Expert Opin Orphan Drugs 2013;1 (8):66173.

2. Gnyszka A, Jastrzebski Z, Flis S. DNA Methyltransferase Inhibitors and Their Emerging Role in Epigenetic Therapy of Cancer. Anticancer Res 2013;33(8):2989-96.

3. Singh V, Sharma P, Capalash N. DNA Methyltransferase-1 Inhibitors as Epigenetic Therapy for Cancer. Curr Cancer Drug Targets 2013;13(4):379-99.

4. Stresemann C, Lyko F. Modes of action of the DNA methyltransferase inhibitors azacytidine and decitabine. Int J Cancer 2008;123(1):8-13.

5. Rogstad DK, Herring JL, Theruvathu JA, Burdzy A, Perry CC, Neidigh JW, Sowers LC. Chemical Decomposition of 5-Aza-2 '-deoxycytidine (Decitabine): Kinetic Analyses and Identification of Products by NMR, HPLC, and Mass Spectrometry. Chem Res Toxicol 2009;22(6):1194204.

6. Kim JK, Kim HJ, Chung JY, Lee JH, Young SB, Kim YH. Natural and synthetic biomaterials for controlled drug delivery. Arch Pharm Res 2014;37(1):60-8.

7. Doppalapudi S, Jain A, Khan W, Domb AJ. Biodegradable polymersan overview. Polym Adv Technol 2014;25(5):427-35.

8. Parent M, Nouvel C, Koerber M, Sapin A, Maincent P, Boudier A PLGA in situ implants formed by phase inversion: Critical physicochemical parameters to modulate drug release. J Control Release 2013;172(1):292-304.

9. Lu CC, Wang XJ, Wu GL, Wang JJ, Wang YN, Gao H, Ma JB. An injectable and biodegradable hydrogel based on poly(alpha,beta-aspartic acid) derivatives for localized drug delivery. J Biomed Mater Res Part A 2014;102(3):628-38.

10. Jain JP, Modi S, Domb AJ, Kumar N. Role of polyanhydrides as localized drug carriers. J Control Release 2005;103(3):541-63.

11. Jain JP, Chitkara D, Kumar N. Polyanhydrides as localized drug delivery carrier: an update. Expert Opin Drug Deliv 2008;5(8):889-907.

12. Pereira DY, Yip AT, Lee BS, Kamei DT. Modeling Mass Transfer from Carmustine-Loaded Polymeric Implants for Malignant Gliomas. J Lab Autom 2014;19(1):19-34.

13. Casalini T, Perale G. Types of bioresorbable polymers for medical applications. In: Jenkins M, Stamboulis A, editors. Durability and Reliability of Medical Polymers. Cambridge: Woodhead Publ Ltd; 2002. p. 3-29.

14. Teomim D, Domb AJ. Fatty acid terminated polyanhydrides. J Polym Sci, Part A: Polym Chem 1999;37(16):3337-44.

15. Llinàs A, Burley JC, Box KJ, Glen RC, Goodman JM. Diclofenac solubility: Independent determination of the intrinsic solubility of three crystal forms. J Med Chem 2007;50(5):979-83.

16. Kipper MJ, Shen E, Determan A, Narasimhan B. Design of an injectable system based on bioerodible polyanhydride microspheres for sustained drug delivery. Biomaterials 2002;23(22):4405-12.

17. Brus J. Heating of samples induced by fast magic-angle spinning. Solid State Nucl Magn Reson 2000;16(3):151-60.

18. Okochi-Takada E, Ichimura S, Kaneda A, Sugimura T, Ushijima T. Establishment of a detection system for demethylating agents using an endogenous promoter CpG island. Mutat Res 2004;568(2):187-94.

19. Policianova O, Brus J, Hruby M, Urbanova M, Zhigunov A, Kredatusova J, Kobera L. Structural Diversity of Solid Dispersions of Acetylsalicylic Acid As Seen by Solid-State NMR. Mol Pharmaceutics 2014;11(2):516-30.

20. Urbanova M, Brus J, Sedenkova I, Policianova O, Kobera L. Characterization of solid polymer dispersions of active pharmaceutical ingredients by F-19 MAS NMR and factor analysis. Spectrochim Acta A Mol Biomol Spectrosc 2013;100:59-66. 